\title{
Fatores de risco relacionados a pré-eclâmpsia: uma revisão integrativa da literatura
}

Risk factors related to pre-eclampsia: an integrative review of the literature

Factores de riesgo relacionados a la pre-eclámpsia: una revista integrativa de la literatura

Ticianne da Cunha Soares ${ }^{1 *}$, Luana Carla Braga Santana ${ }^{1}$, Tamires da Cunha Soares ${ }^{1}$, João Caio Silva Castro Ferreira ${ }^{1}$, Ariella de Carvalho Luz', Maria de Fatima Sousa Barros Vilarinho', Jéssica Denise Vieira Leal' ${ }^{1}$, Victor Alves de Oliveira ${ }^{1}$, Joice Rodrigues de Almeida ${ }^{1}$, Vitória Silva de Araújo ${ }^{1}$, Renato Felipe de Andrade'.

\section{RESUMO}

Objetivo: Analisar os fatores de risco relacionados à pré-eclâmpsia. Método: Revisão integrativa da literatura que pesquisou estudos que abordassem a temática dos fatores de risco relacionados a PE. Foram inclusos na revisão todos os artigos em inglês e português, publicados entre o período de 2005 a 2018. Resultados: Pôde-se observar que os fatores de risco para PE encontrados: fatores sociodemográficos, história familiar de PE, história pessoal de PE, IMC pré-gestacional elevado, ganho de peso gestacional acima do esperado, extremos de idade reprodutiva, presença de doenças crônicas, raça negra e primiparidade. Considerações finais: Acredita-se que novas publicações sobre estes fatores são úteis por auxiliar na identificação das mulheres com maior probabilidade de desenvolvê-la, e futuramente, investir em pré-natal adequado, que pode contribuir na redução dessa realidade.

Descritores: Fatores de risco, Pré-eclâmpsia, Gravidez.

\begin{abstract}
Objective: To analyze the risk factors related to pre-eclampsia. Method: Integrative review of the literature that investigated studies that addressed the theme of risk factors related to PE. All articles in English and Portuguese published between 2005 and 2018. Results: It was observed that the risk factors for PE found: sociodemographic factors, family history of PE, personal history of PE, elevated pre-gestational BMI, gestational weight gain above expectations, extremes of reproductive age, presence of chronic diseases, black race and primiparity. Final Remarks: It is believed that new publications on these factors are useful to help identify the women most likely to develop it, and in the future, invest in adequate prenatal care, which may contribute to the reduction of in reducing this reality.
\end{abstract}

Descriptors: Risk factors, Pre-Eclampsia, Pregnancy.

\section{RESUMEN}

Objetivo: Analizar factores de riesgo relacionados con la preeclampsia. Método: Revisión integrativa de la literatura que investigó estudios que abordasen la temática de los factores de riesgo relacionados con la PE. Se incluyeron en la revisión todos los artículos en Inglés y portugués, publicados entre el período de 2005 a 2018. Resultados: Se observó que los factores de riesgo de PE encontrados: factores sociodemográficos, antecedentes familiares de PE, PE historia personal, índice de masa corporal en el caso de las mujeres embarazadas, las mujeres embarazadas y las mujeres. Consideraciones Finales: Se cree que nuevas

${ }^{1}$ Universidade Federal do Piauí. Picos, PI, Brasil. *E-mail: ticiannesoares@outlook.com 
publicaciones sobre estos factores son útiles por auxiliar en la identificación de las mujeres con mayor probabilidad de desarrollarla, y en el futuro, invertir en prenatal adecuado, que puede contribuir en la reducción de esa realidad.

Descriptores: Factores de riesgo, La preeclampsia, Embarazo.

\section{INTRODUÇÃO}

A Síndrome Hipertensiva Específica da Gestação (SHEG) é um dos grandes desafios da saúde pública no mundo inteiro, uma vez que se configura como a primeira causa de morbimortalidade materno-fetal no Brasil e a terceira causa no mundo (BRASIL, 2012a). Os agravos da hipertensão arterial sistêmica (HAS) são os que mais comumente acometem mulheres no período gestacional, entre 5 a $10 \%$, e se classificam em: hipertensão arterial crônica, hipertensão gestacional, pré-eclâmpsia (PE), eclampsia, e PE superposta à HAS crônica (BRASIL, 2012b).

Dos distúrbios hipertensivos, a PE é a que mais atinge gestantes e de 10 a $15 \%$ das mortes maternas mundiais estão ligadas a ela ou a eclâmpsia. Esta, se caracteriza pelo o aumento da pressão arterial que acontece após a $20^{\circ}$ semana de gestação acompanhado de proteinúria, com desaparecimento até $12^{\circ}$ semanas pós-parto, podendo haver ou não edema, já a eclâmpsia há a presença de convulsões tônico crônicas generalizadas em mulheres com quaisquer dos quadros hipertensivos supracitados, não causadas por epilepsia ou outra patologia convulsiva qualquer, podendo ocorrer na gravidez, parto ou até dez dias de puerpério (BRASIL, 2010). Quando não há proteinúria, os sinais que consolidam a possível PE são quando a hipertensão aparece associada a cefaleia, distúrbios visuais, dor abdominal, plaquetopenia e aumento de enzimas hepáticas (WORLD HEALTH ORGANIZATION, 2005). Acomete 2 a $8 \%$ das gestantes e em pacientes com história preexistente da patologia, essa taxa sobe para 18\% (MONTENEGRO CAB, et al., 2008).

Os casos de PE estão diretamente associados ao risco crescente de acontecimentos desfavoráveis como descolamento de placenta que pode provocar o nascimento prematuro e aumento dos riscos logo ao nascer, hemorragia cerebral, insuficiência renal aguda e outros (GRUSLIN A e LEMYRE B, 2011) e resultados perinatais inapropriados, como macrossomia fetal, baixo peso ao nascer, síndrome de aspiração meconial, baixa Apgar de 1 e 5 minutos e outros (OLIVEIRA CA, et al., 2006).

Diversos fatores de risco relacionados a um maior risco de desenvolvimento da PE são descritos em estudos, dentre eles, extremos de idade reprodutiva, ganho de peso excessivo, nutrição pré e/ou gestacional inadequado, histórico familiar e/ou pessoal de PE, condições socioeconômicas desfavoráveis, presença de doenças crônicas, raça negra e outros (OLIVEIRA ACM, et al., 2016).

Alguns sinais podem caracterizar a PE, como presença de edema, especialmente na face, ao redor dos olhos, mãos e membros inferiores, ganho ponderal intenso, náuseas e/ou vômitos, dor abdominal com irradiação para os membros superiores, cefaleia e complicações visuais, como a visão turva. Entretanto, por vezes, essa condição se instala e evolui totalmente silenciosa, sem sinais ou sintomas (OMS, 2014).

Tendo em vista as particularidades, a taxa de morbimortalidade e a ocorrência de casos de pré-eclâmpsia, evidencia-se a importância de identificar os possíveis fatores de risco a essa relacionado, além de amplificar os estudos acerca dessa temática. Justifica-se este estudo a partir da necessidade de conhecer os fatores de risco que predispõem a ocorrência da PE. Assim, este estudo teve como questão de pesquisa: Quais os fatores de risco mais comumente associados a préeclâmpsia? Neste sentido, determinou-se como objetivo analisar os fatores de risco relacionados à préeclâmpsia. 


\section{MÉTODO}

Trata-se de uma revisão integrativa da literatura que pesquisou estudos que abordassem a temática dos fatores de risco relacionados a PE. Este método tem por finalidade condensar as pesquisas publicadas, a fim de obter novos desfechos a partir de um tema de interesse (WHITTEMORE R e KNAFL K, 2005). Os resultados de uma revisão integrativa possibilitam gerar novas discussões e reflexões sobre a temática pesquisada, identificando déficits no conhecimento que necessitam de outras investigações e realização de pesquisas futuras, além de traçar a tomada de decisões e melhoria da prática clínica (MENDES KDS, et al., 2008).

$\mathrm{Na}$ instrumentalização desta revisão, utilizou-se das seguintes etapas: delimitação do tema e o estabelecimento da questão de pesquisa; determinação dos critérios de inclusão e exclusão e a pesquisa na literatura; indicação das informações a serem retiradas dos artigos escolhidos e classificação dos estudos; avaliação dos estudos que se enquadrem na revisão integrativa; interpretação dos resultados; exposição da revisão e a sinopse do estudo investigações e realização de pesquisas futuras, além de traçar a tomada de decisões e melhoria da prática clínica (MENDES KDS, et al., 2008).

A seleção dos artigos foi realizada entre os meses de abril a junho de 2018, por meio da busca nas bases de dados informatizados, sendo estas Biblioteca Virtual em Saúde (BVS) e Scientific Electronic Library Online (SCIELO). Na BVS, consta um segmento específico que dá acesso a outras bases de dados das quais utilizou-se Medical Literature Analysis and Retrieval System Online (MEDLINE) e Literatura Latino-Americana e do Caribe em Ciências da Saúde (LILACS).

A pesquisa foi realizada através de formulário avançado, utilizando os descritores de saúde: Fatores de risco; Pré-eclâmpsia; Gestantes, sendo o principal termo aplicado "pré-eclâmpsia" combinado com os outros descritores citados. Foram inclusos na revisão todos os artigos em inglês e português, publicados entre o período de 2005 a 2018. Ademais, considerou-se inclusos os artigos originais que respondessem à questão de pesquisa.

Por outro lado, foram excluídos os trabalhos que não estavam disponíveis na íntegra e/ou que não se enquadrem nos critérios de inclusão anteriormente citados, uma vez que estes fatores dificultariam a extração de informações confiáveis e relevantes para a investigação dos fatores e desfecho estudados. Ressalta-se que os artigos repetidos nas bases de dados foram considerados apenas uma vez.

A primeira fase da seleção dos artigos se deu por meio da análise do título e seguidamente, dos resumos. Em seguida, os estudos foram analisados por meio de quatro leituras, sendo estas: exploratória, seletiva, analítica e interpretativa (GIL AC, 2002). Durante a leitura exploratória, foram analisados os objetivos de pesquisa dos artigos encontrados. A segunda leitura trouxe a seleção de artigos que realmente fossem relevantes à pesquisa, com base nos critérios de inclusão e exclusão. Posteriormente, foi realizada a leitura analítica, onde os dados foram analisados, organizados e sumariados. Por fim, a leitura interpretativa relacionando as afirmações do autor com os objetivos do presente estudo.

Mediante a leitura dos artigos inclusos, com o objetivo de organizar a coleta e análise dos artigos que se encaixem nos critérios de inclusão, adotou-se um instrumento para agrupar e resumir o conteúdo-chave das publicações, composta por título, objetivo, delineamento, base de dados, ano de publicação, faixa etária, renda familiar, estado civil, grau de escolaridade, raça e se já houveram outras gestações.

Após a busca realizada, encontrou-se um total de 121 artigos nas duas bases de dados (19 na SCIELO e 102 na BVS), dos quais, após a filtragem, 114 não se enquadravam nos critérios de inclusão supracitados. Consequentemente, finalizou-se a busca com 7 estudos para análise. Afim de melhor ilustrar a busca e a exclusão dos artigos, criou-se um fluxograma de representação do processo de seleção dos artigos inclusos na revisão. 
Estratégia de busca: ("fatores de risco") AND ("pré-eclâmpsia") AND ("gravidez") [Descritor do assunto] and "INGLÊS" or "PORTUGUÊS" [Idioma] and "2005" or "2006" or "2007" or "2008" or "2009" or "2010" or "2011" or "2012" or "2013" or "2014" or "2015" or "2016" or "2017" or "2018" [ano de publicação].

Figura 1 - Fluxograma de representação das buscas realizadas nas bases de dados e artigos selecionados em abril e maio de 2018.
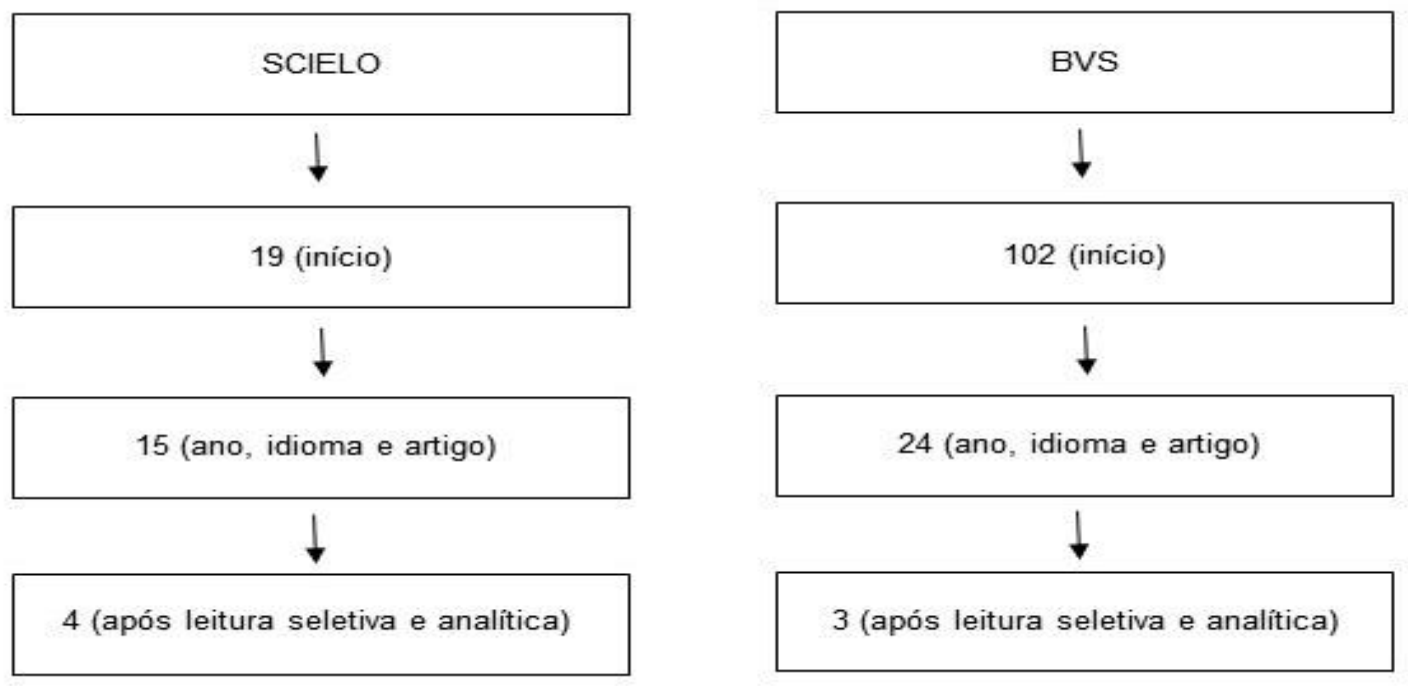

Fonte: Próprio autor, 2018.

\section{RESULTADOS E DISCUSSÃO}

No Quadro 1 são apresentados os artigos incluídos no estudo, conforme os critérios estabelecidos. Ressalta-se que os artigos encontrados foram publicados entre os anos de 2008 e 2017.

Quadro 1 - Artigos selecionados nas bases de dados SCIELO e BVS. Pimenteiras, PI, 2018.

\begin{tabular}{|c|c|c|c|}
\hline Cod & Título & Base & Ano \\
\hline A1 & Risk factors for hypertensive disorders of pregnancy in southern Brasil & SCIELO & 2011 \\
\hline A2 & $\begin{array}{l}\text { Fatores de risco para síndrome hipertensiva específica da gestação entre } \\
\text { mulheres hospitalizadas com pré-eclâmpsia }\end{array}$ & BVS & 2010 \\
\hline A3 & $\begin{array}{l}\text { Perfil de risco gestacional e metabólico no serviço de pré-natal de maternidade } \\
\text { pública do Nordeste do Brasil }\end{array}$ & SCIELO & 2012 \\
\hline A4 & $\begin{array}{l}\text { Estudo dos principais fatores de risco maternos nas síndromes hipertensivas } \\
\text { da gestação }\end{array}$ & BVS & 2008 \\
\hline A5 & $\begin{array}{l}\text { Maternal Factors and Adverse Perinatal Outcomes in Women with } \\
\text { Preeclampsia in Maceió, Alagoas }\end{array}$ & BVS & 2016 \\
\hline A6 & $\begin{array}{l}\text { Prevalência de síndromes hipertensivas gestacionais em usuárias de um } \\
\text { hospital no Sul do Brasil }\end{array}$ & SCIELO & 2017 \\
\hline A7 & Gestational diabetes and pre-eclampsia: common antecedentes? & SCIELO & 2008 \\
\hline
\end{tabular}

Fonte: Próprio autor, 2018. 
Dentre os artigos incluídos nessa análise, no tocante à abordagem, dois estudos eram caso-controle, dois transversais, dois de coorte e um longitudinal. Quanto ao país de origem do estudo, os sete foram realizados no Brasil, prevalecendo o estado do Rio Grande do Sul com três estudos, os estados do Ceará, Alagoas, Bahia e Goiás com um estudo cada. No que se refere ao ano de publicação, 2008 teve dois estudos e 2010, 2011, 2012, 2016, 2017 um estudo cada. No tocante ao objetivo desta revisão, pôde-se observar que os fatores de risco para PE encontrados foram:

Quadro 2 - Fatores de risco para PE encontrados nos artigos selecionados. Pimenteiras, PI, 2018.

\begin{tabular}{|l|l|}
\hline Fatores de ricos & Código do artigo \\
\hline Fatores sociodemográficos & A1, A2, A3, A4, A5, A6 \\
\hline História familiar de PE & A1, A2, A5 \\
\hline História pessoal de PE & A1, A2, A4, A5 \\
\hline IMC pré-gestacional elevado & A3, A4, A7 \\
\hline Ganho de peso gestacional acima do esperado & A1, A3 \\
\hline Extremos de idade reprodutiva & A2, A5, A7 \\
\hline Presença de doenças crônicas & A1, A2 \\
\hline Raça negra & A3, A4, A5 \\
\hline Primiparidade & A2, A4 \\
\hline
\end{tabular}

Fonte: Próprio autor, 2018.

Ressalta-se que os periódicos com mais publicações sobre essa temática foi o Arquivos Brasileiros de Cardiologia (A4 e A5), seguindo-se a Revista da Associação Médica Brasileira (A1), Cogitare Enfermagem (A2), Revista Brasileira de Ginecologia e Obstetrícia (A3), Revista Cuidarte (A6) e Arquivos Brasileiros de Endocrinologia e Metabologia (A7).

Quanto ao perfil sociodemográfico das gestantes acometidas pela PE, em estudo de Dalmáz CA, et al. (2011), 73\% é da cor branca e a idade média era de 29 anos (13 a 48 anos). Para Moura ERF, et al. (2010), a I caracterização da amostra se deu de forma mais detalhada, a idade varia entre 15 e 43 anos (a maior parte, $47,5 \%$, entre 15 e 21 anos), quanto ao grau de escolaridade, $50 \%$ estão entre ensino fundamental completo e ensino médio completo, quanto a ocupação, $60 \%$ trabalha em casa, ou seja, tem as atividades do lar por ocupação e a renda familiar de $75 \%$ é entre 1-2 salários mínimos. Na população utilizada no estudo de Santos EMF, et al. (2012), a média de idade foi 26 anos (variando entre 13 a 42 anos), com predominância da cor parda, 59,8\%, 51,8\% com segundo grau completo, 51\% desenvolve atividade remunerada e a maioria convive em união estável.

No estudo de Assis TR, et al. (2008), a idade média do grupo de PE foi $23,3 \pm 5,8,46,3 \%$ não concluiu o segundo grau, a proporção entre casadas e solteiras é igual e $64,9 \%$ tinha renda familiar de um a três salários mínimos, vale ressaltar que nesse estudo em específico, não se fez associação entre as variáveis citadas com a ocorrência de PE e com outras SHEG quando comparadas com o grupo controle, o que é associado a homogeneidade da população estudada. Em estudo de Oliveira CA, et al. (2016), a faixa etária variou entre $25,8 \pm 6,7$ anos ( $\leq 19 \mathrm{a} \geq 35$ anos), das quais $17,8 \%$ eram adolescentes, $43,3 \%$ tinham baixa escolaridade, $30,0 \%$ baixa renda, $16,7 \%$ relatou a cor da pele preta. $92 \%$ da população estudada no artigo de Kerber GF e Melere C (2017) era da cor branca e a média de idade se encontrava aproximadamente 29 $\pm 5,7$ anos.

A ligação entre condições socioeconômicas e o aumento dos casos de PE se faz com base no fato da possibilidade dessa condição ser associada a episódios de estresse, facilmente atrelados a necessidades 
básicas não supridas e inadequado estado nutricional (MOURA ERF, et al., 2010). Alguns estudos também apontam os fatores sociodemográficos da população com PE, idade entre 14 e 50 anos de idade (média $26,1 \%), 34,8 \%$ da cor parda, $41,6 \%$ ensino fundamental completo e $35,6 \%$ tinham ocupação não registrada e antecedentes familiares e pessoais (AMORIM FCM, et al., 2017), $26 \pm 6,3$ anos, baixo nível de instrução e baixos rendimentos (AZEVEDO DV, et al., 2009), 18 a 26 anos, 41,66\% tem ensino fundamental completo, $58,33 \%$ e a renda variava entre 100 a 700 reais (CUNHA KJB, et al., 2007) idade entre 18 e 35 anos, escolaridade de $82,1 \%$ entre fundamental e médio e renda menor que 3 salários mínimos (SOUZA NL, et al., 2007).

Os dados supracitados também demonstram que a idade da mãe configura como fator desencadeador de algumas intercorrências no período gestacional, gestação de adolescente, tal qual a que ocorre em idade avançada, são tidas como risco aumentado para PE (MOURA ERF, et al., 2010); (SANTOS EMF, et al., 2012); (ASSIS TR, et al., 2008); (OLIVEIRA ACM, et al., 2016); (WENDLAND EMR, et al., 2008). A raça também é apontada como fator de risco por (SANTOS EMF, et al., 2012); (ASSIS TR, et al., 2008); (OLIVEIRA ACM, et al., 2016) pessoas que tem a cor de pele preta aparentemente apresentam uma falha hereditária na captação celular e transporte renal de cálcio e sódio, que pode ser concedido a um gene poupador de sódio que propicia o influxo celular de sódio e o efluxo de cálcio, facilitando o desenvolvimento de hipertensão (JOHNSON III EF e WRIGHT JT JR., 2005).

O fator história familiar de PE apresenta-se em estudo de Dalmáz CA, et al. (2011) se faz presente em $44 \%$ do grupo que continha essa patologia e em $20 \%$ do grupo controle tinha esse histórico, já no estudo de Moura ERF, et al. (2010), do grupo pesquisado $30 \%$ além de $62,5 \%$ de hipertensão e $28,9 \%$ (OLIVEIRA ACM, et al., 2016). Os casos de PE ocorrem mais frequentemente em pacientes que tenham predisposição genética (FREITAS F, et al., 2006).

Como já mencionado anteriormente, a história pessoal de PE se configura como fator de risco para desenvolvimento dessa condição e de alguma outra SHEG em gestações posteriores (AMORIM FCM, et al., 2017), esse dado se fez presente em $57 \%$ do público pesquisado por (DALMÁZ CA, et al., 2011), no estudo de Moura et al. (2010), das dezenove multigestas, que compreende $47,5 \%$ da população analisada, doze $(63,1 \%)$ afirmaram PE na gestação anterior. Em estudo de Assis TR, et al., (2008), 83,6\% e 38,9\% (OLIVEIRA ACM, et al., 2016). Pacientes que desenvolvem a PE apresentam maior risco de desenvolver a patologia nas gestações futuras, e por vezes, apresentam também histórico familiar de PE, o que remete a fatores genéticos, entretanto, as questões sobre a razão genética da PE não são totalmente comprobatórias (WILLIAMS PJ e BROUGHTON PIPKN FB, 2011).

Em Santos EMF, et al. (2012); Assis TR, et al. (2008); (WENDLAND EMR, et al., 2008), o IMC prégestacional se encontrava elevado $34,6 \%$ (SANTOS et al., 2010), 90,2\% da população com PE estudada era obesa (ASSIS TR, et al., 2008) e $32.9 \pm 6.1 \%$ (WENDLAND EMR, et al., 2008). O ganho de peso acima do esperado 32,9 \pm 6,1\% (DALMÁZ CA, et al., 2011), 45,5\% (SANTOS EMF, et al., 2010). Alguns maus hábitos alimentares foram relatados (MOURA ERF, et al., 2010), o principal grupo de alimentos relatado foi o dos carboidratos, como arroz e massas em geral e pão francês eram consumidos por $85 \%$ ou mais das grávidas, seguido por lipídeos, por 77,5\%, em contrapartida, apresentou-se carência em alguns alimentos, como o leite, carne bovina, frango e feijão, que são ricos em proteínas, são ausentes nas dietas das gestantes em $90 \%, 77,5 \%, 75 \%$ e $52,5 \%$, respectivamente, além do uso abusivo de sal, relatado por $55 \%$ das gestantes.

Os carboidratos são fonte de energia para o corpo, contudo, se consumido excessivamente, o que excede é transformado em tecido adiposo, que provoca o ganho exagerado de peso, podendo levar a obesidade e expondo a mulher a maior chance de desenvolvimento de SHEG. Os lipídeos de origem animal, como as frituras relatadas por Moura ERF, et al. (2010), são ricos em colesterol e gordura saturada, o que propicia o ganho de peso. O leite, é fonte importante de cálcio e vitamina B6, assim como os sucos de fruta (PAIVA ES, 2006), que só foram relatados respectivamente, por 10\% e 12,5\% (MOURA ERF, et al., 2010). 
A primiparidade foi apontada por 27\% (DALMÁZ CA, et al., 2011), 52,5\% (MOURA ERF, et al., 2010) e 54\% (ASSIS TR, et al., 2008). A PE é mais comum em primíparas (LOWDERMILK DL, et al., 2002), pois a primeira gestação é geralmente ligada a uma situação de estresse atípico, e por este motivo, se configura como um fator de risco (TAKIUTI NH e KAHHALE S , 2001).

Com relação a prevenção da $\mathrm{PE}$, na prática clínica, as alternativas recomendáveis e tidas como efetivas, são somente o uso de cálcio (carbonato de cálcio, 1.000-2.000 mg/dia) e aspirina em baixa dose (50-150 $\mathrm{mg} / \mathrm{dia}$ ) (FEBRASGO, 2017). Quanto ao tratamento, inicialmente, consiste na redução da pressão sanguínea materna e aumento do fluxo sanguíneo placentário (FERRÃO MHL, et al., 2006). Nestes casos, as drogas utilizadas como anti-hipertensivos são a hidralazina e a metildopa, que agem estimulando o relaxamento do músculo liso das arteríolas periféricas e a diminuição da resistência vascular. Como rastreamento, observa-se os fatores de risco e deve-se ter maior atenção no pré-natal, objetivando o diagnóstico de PE o mais precocemente possível (FEBRASGO, 2017).

Salienta-se que para a realização desta análise foram consultados apenas artigos disponíveis na íntegra e online e utilizadas somente duas bases de dados, assim sendo, nela pode conter limitações no tocante aos estudos a respeito da temática abordada.

\section{CONSIDERAÇÕES FINAIS}

A PE é uma patologia de etiologia ainda desconhecida e, como supracitado, acomete mulheres no mundo inteiro, com diversos fatores de risco associados/predisponentes e que se não tratada corretamente, pode gerar desfechos desfavoráveis para mãe, $\mathrm{RN}$ ou ambos, para tanto, possivelmente a melhor solução para a mesma é a detecção precoce. Fatores como os sociodemográficos e primiparidade, se configuram como estressores, além disso, somados a predisposição genética, presença de doenças crônicas, extremos de idade e maus hábitos alimentares, se posicionam como risco a PE. Acredita-se ainda que novas publicações sobre estes fatores são úteis por auxiliar na identificação das mulheres com maior probabilidade de desenvolvê-la, e futuramente, investir em pré-natal adequado, que pode contribuir na redução dessa realidade.

\section{REFERÊNCIAS}

1. AMORIM FCM, et al. Perfil de gestantes com pré-eclâmpsia. Rev enferm UFPE online. 2017;11(4):1574-83.

2. ASSIS TR, et al. Estudo dos principais fatores de risco maternos nas síndromes hipertensivas da gestação. Arq. Bras. Cardiol. 2008;91(1):11-7.

3. AZEVEDO DV, et al. Percepções e sentimentos de gestantes e puérperas sobre a pré-eclâmpsia. Rev. Salud pública. 2009;11(3)347-58.

4. BRASIL. Ministério da Saúde. Atenção ao pré-natal de baixo risco. 1ํe ed. Brasília: Ministério da Saúde, 2012. (Cadernos de Atenção Básica, 32)

5. BRASIL. Ministério da Saúde. Coordenação Geral de Informações e Análise Epidemiológica, Secretaria de Vigilância em Saúde. Saúde Brasil 2011 uma análise da situação de saúde e a vigilância da saúde da mulher. 1ำed. Brasília: Ministério da Saúde, 2012.

6. BRASIL. Ministério da Saúde. Secretaria de Políticas de Saúde. Gestação de alto risco. Manual técnico. $3^{\underline{a}}$ ed. Brasília: Ministério da Saúde. 2010.

7. CUNHA KJB, et al. Assistência de enfermagem na opinião das mulheres com pré-eclâmpsia. Esc Anna Nery R Enferm. 2007;11(2):254-60.

8. DALMÁZ CA, et al. Risk factors for hypertensive disorders of pregnancy in southern Brazil. Rev. Assoc. Med. Bras. 2011;57(6):692-96.

9. Federação Brasileira de Ginecologia e Obstetrícia (FEBRASGO). Pré-eclâmpsia. n. 8, 2017. 21-4 p.

10. FERRÃO MHL, et al. Efetividade do tratamento de gestantes hipertensas. Rev Assoc Med Bras. 2006; 52(6): 390-4.

11. FREITAS F, et al. Rotinas em obstetrícia. 5a ed. Porto Alegre: Artes Médicas; 2006.

12. GIL AC. Como elaborar projetos de pesquisa. 4 ed. Editora Atlas; 2002. 176 p. 
13. GRUSLIN A, LEMYRE B. Pré-eclâmpsia: avaliação fetal e desfechos neonatais. Melhor prática Res Clin Obstet Gynaecol. 2011; 25(4): 491-507.

14. JOHNSON III EF, WRIGHT JT JR. Management of hypertension in black populations. In: Oparil S, Weber MA. Hypertension. 2nd. ed. New York: Elsevier; 2005. p. 587-95.

15. KERBER GF, MELERE C. Prevalência de síndromes hipertensivas gestacionais em usuárias de um hospital no Sul do Brasil. Rev Cuid. 2017;8(3):1899-06.

16. LOWDERMILK DL, et al. O cuidado em enfermagem materna. 5aㅡ ed. São Paulo: Artmed; 2002.

17. MENDES KDS, et al. Revisão integrativa: método de pesquisa para incorporação de evidências na saúde e na enfermagem. Texto Contexto Enferm. 2008;17(4): 758-64.

18. MONTENEGRO CAB, et al. - Obstetrícia fundamental. 11aㅡ ed. Rio de Janeiro: Guanabara Koogan; 2008; 33974.

19. MOURA ERF, et al. Fatores de risco para síndrome hipertensiva específica da gestação entre mulheres hospitalizadas com pré-eclâmpsia. Cogitare Enferm. 2010;15(2): 250-5.

20. OLIVEIRA ACM, et al. Maternal Factors and Adverse Perinatal Outcomes in Women with Preeclampsia in Maceió, Alagoas. Arq. Bras. Cardiol. 2016;106(2):113-20.

21. OLIVEIRA CA, et al. Síndromes hipertensivas da gestação e repercussões perinatais. Rev Bras Saúde Matern Infant. 2006; 6(1): 93-8.

22. ORGANIZAÇÃO MUNDIAL DA SAÚDE (OMS). Recomendações da OMS para a prevenção e tratamento da pré-eclâmpsia e eclâmpsia. Brasília: OMS. 2014.

23. PAIVA ES. Como deve ser a alimentação da gestante hipertensa. Jornal O Norte [Internet] João Pessoa; 2006.

24. SANTOS EMF, et al. Perfil de risco gestacional e metabólico no serviço de pré-natal de maternidade pública do Nordeste do Brasil. Ver. Ginecol. Obstet. 2012;34(3):102-6.

25. SOUZA NL, et al. Percepção materna com o nascimento prematuro e vivência da gravidez com pré-eclâmpsia. Rev Saúde Pública. 2007;41(5):704-10.

26. TAKIUTI NH, KAHHALE S. Estresse e pré-eclâmpsia. Rev Assoc Med Bras. 2001;47(2):88-9.

27. WENDLAND EMR, et al. Gestational diabetes and pre-eclampsia: common antecedentes? Arq Bras Endocrinol. 2008;52(6):975-84.

28. WHITTEMORE R, KNAFL K. The integrative review: updated methodology. J Adv Nurs. 2005; 52(5): 546-53.

29. WILLIAMS PJ, BROUGHTON PIPKN FB. The genetics of pre-eclampsia and other hypertensive disorders of pregnancy. Best Pract Res Clin Obstet Gynaecol. 2011;25(4):405-17.

30. WORLD HEALTH ORGANIZATION. The World Health Report 2005 - make every mother and child count. Geneva: World Health Organization. 2005. 